JOURNAL DE PHYSIQUE IV

Colloque C3, supplément au Journal de Physique III, Volume 2, décembre 1992

\title{
Les aciers électriques pour transformateurs et machines tournantes. Situation actuelle et perspectives
}

\author{
J.-C. BAVAY et J. VERDUN*
}

UGINE S.A., Centre de Recherche d'Isbergues, BP. 15, 62330 Isbergues, France

"G.I.E. USINOR Aciers Electriques Ile de France, 4 Place de la Pyramide, 92070 Paris la Défense cedex 33, France

The total losses improvement of non-oriented and grain-oriented electrical steels is described on the basis of the effects of $S i$ and $A l$ contents, strip thickness, impurities, grain diameter, texture, stress induced by insulation coating and laser treatment. Non-oriented electrical steels with more isotropic magnetic properties and higher magnetic induction due to the ideal texture $\{100\}\langle 0 \mathrm{vw}\rangle$, reduced thickness, lower contents of impurities in connection with the progress of clean steel producing technology and optimum grain size, are viewed as promising core materials for rotating machines. A guiding principe for developing lower-loss grain-oriented electrical steels is to increase the degree of $\{110\}\langle 100\rangle$ orientation and the silicon content and to decrease the thickness and the grain size in association with a smoother surface and an artificial domain refining technique. The [100][100] double-oriented texture appears the ideal texture for grain-oriented electrical steels used in stacked transformers.

\section{Introduction}

L'aimantation des substances ferromagnétiques constitue encore aujourd'hui la seule source d'induction magnétique économiquement acceptable. De ce fait, la tôle magnétique à base de fer allié au silicium et à l'aluminium est le matériau le plus employé dans les appareillages de production, de transport et de transformation de l'énergie électrique.

Deux grandes familles d'aciers sont offertes pour la construction des circuits magnétiques des équipements électriques dont la conception est basée sur l'action d'un champ magnétique. Les tôles à grains orientés sont utilisées exclusivement pour les circuits magnétiques des machines statiques (transformateurs). C'est la texture de Goss $\{110\}\langle 001\rangle$ qui leur confère des propriétés magnétiques remarquables quand les lignes d'induction sont parallèles à la direction de laminage, elle même très proche de la direction de facile aimantation. Les tôles à grains non orientés sont utilisées pour la construction des machines tournantes 
(générateurs, alternateurs, moteurs).

Les constructeurs électriciens évaluent l'efficacité intrinsèque d'un matériau magnétique à partir de la valeur des pertes totales massiques mesurées à l'induction et à la fréquence de travail. L'éventail des caractéristiques magnétiques des produits disponibles (pertes et aimantation) est analysé sur la base de la composition chimique de l'acier, de la texture du matériau, de la taille de grain, de l'épaisseur de la tôle, du rôle éventuel du revêtement isolant et $d u$ traitement superficiel d'affinement des domaines magnétiques par faisceau laser. Les perspectives d'amélioration des performances magnétiques sont enfin déduites de l'influence de ces différents paramètres.

\section{Influence des éléments d'alliage}

Les performances magnétiques d'un acier électrique sont liées à plusieurs caractéristiques, notamment la valeur de sa polarisation magnétique à saturation, de sa résistivité électrique et de sa constante d'anisotropie magnétocristalline. L'acier électrique idéal est celui qui posséderait une polarisation magnétique à saturation et une résistivité électrique très élevées et, simultanément, une constante d'anisotropie voisine de zéro. Afin de tendre vers cet optimum, il est possible, par addition essentiellement de silicium et d'aluminium, d'améliorer les valeurs de certaines caractéristiques physiques et magnétiques des alliages de fer ainsi obtenus.

Dans la structure cubique centrée du fer, l'aimantation est la plus facile quand la direction du champ d'excitation est parallèle aux directions <001〉 (Fig. 1).

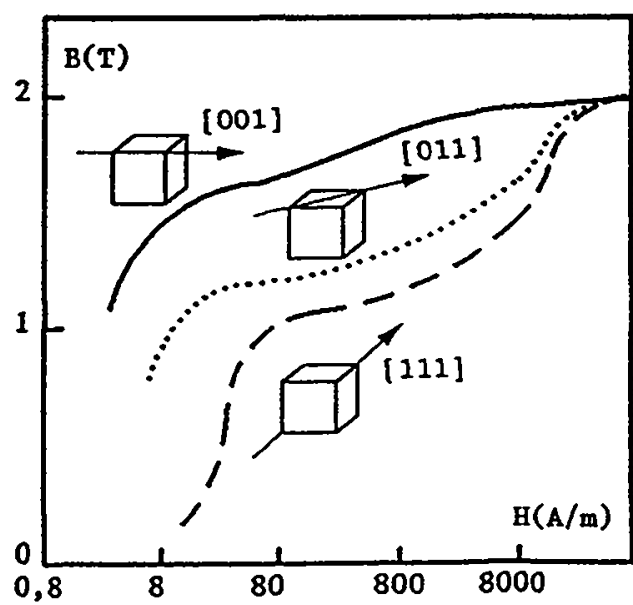

Fig.1 - Induction magnétique $B$ en fonction de la direction du champ $H$ [1]

(Effect of cristallographic direction on magnetisation of a silicon-iron crystal)

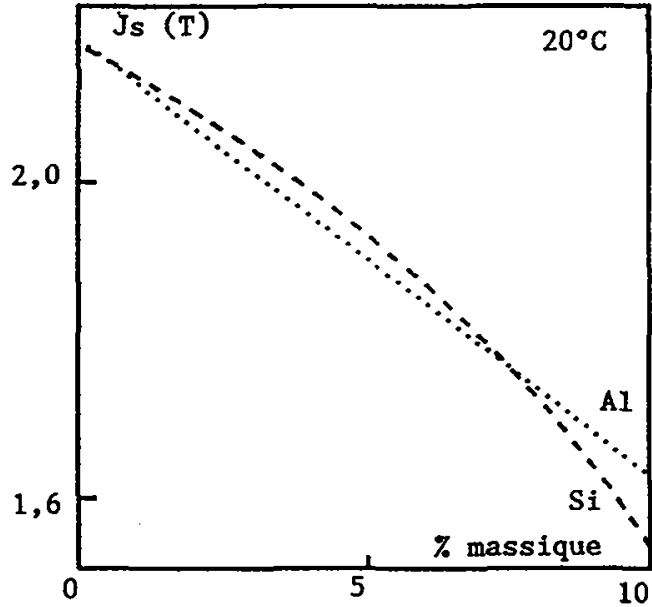

Fig.2 - Polarisation magnétique à saturation Js des alliages Fe-Si et Fe-Al [3] (Saturation induction of $\mathrm{Si}-\mathrm{Fe}$ and $\mathrm{Al}-\mathrm{Fe}$ alloys) 
L'addition au fer de silicium et d'aluminium permet d'abaisser de façon sensible la constante d'anisotropie Kl qui traduit la difficulté avec laquelle les moments magnétiques pivotent vers la direction du champ d'excitation extérieur [4]. Cependant, la polarisation magnétique à saturation décroît en fonction des teneurs en silicium et en aluminium (Fig. 2). Il en résulte une dégradation de la perméabilité de l'acier car pour un même champ d'excitation donné (permettant d'obtenir la saturation du matériau), l'induction acquise est plus faible. En outre, la conductibilité thermique diminue fortement quand la teneur en silicium augmente. Cet effet n'est pas négligeable car les pertes d'énergie sont évacuées à l'extérieur des circuits magnétiques par conduction thermique dans le plan des tôles revêtues ou nor d'un isolant [4].

La présence de silicium et d'aluminium en solution solide dans le fer augmente considérablement la résistivité électrique et par conséquent fait décroître les pertes [4]. En pratique, l'addition de silicium est limitée au maximum à environ 3,3 ; en raison des problèmes de laminage à froid engendrés par la fragilité de l'alliage à haute teneur en silicium [4]. La teneur en aluminium, moins néfaste pour la ductilité, dépasse rarement 0,8 \% en raison de sa grande affinité pour 1 'oxygène et l'azote au cours de l'élaboration et des traitements thermiques.

\section{Aciers électriques à grains non orientés}

Ils contiennent selon les qualités de 0,02 à 3,3 \& de silicium et jusqu'à $0,8 \%$ d'aluminium étant donné 1 'effet bénéfique de ces deux éléments sur les pertes totales massiques $P$ (Fig. 3 ).
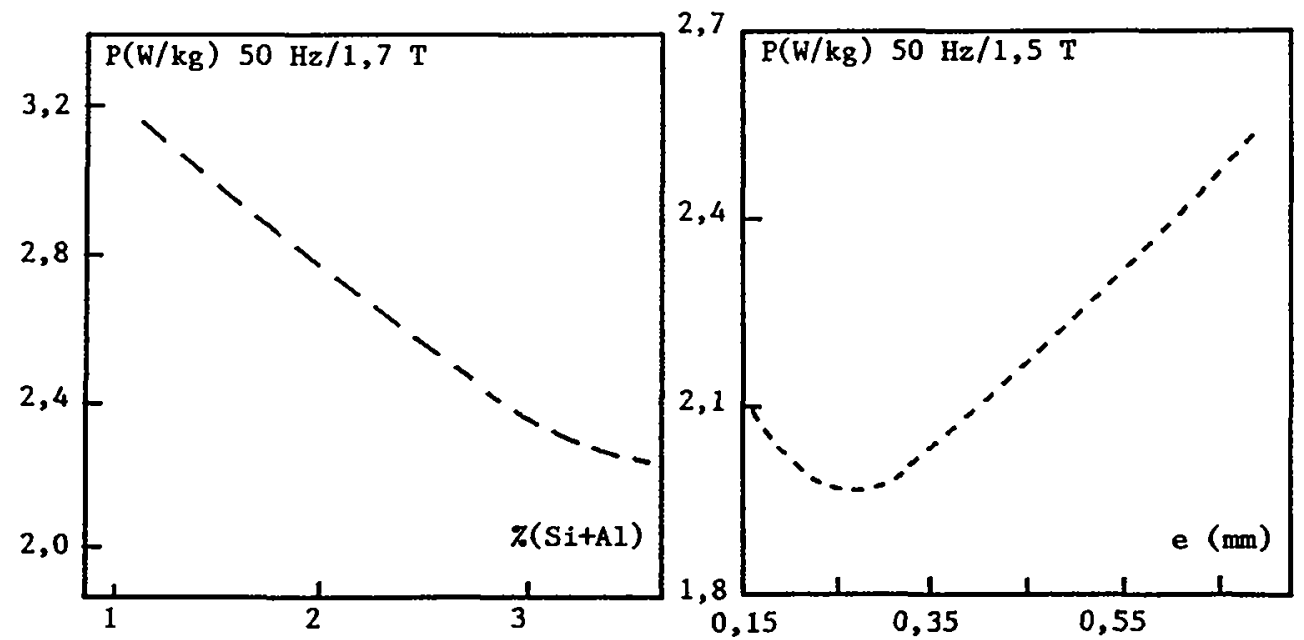

Fig. 3 - Influence du pourcentage massique (Si + Al) d'une tôle de $0,5 \mathrm{~mm}$ d'épaisseur sur les pertes totales massiques [5] (Effect of (Si $+\mathrm{Al})$ content on total losses of $0.5 \mathrm{~mm}$ thick strip)

Fig. 4 - Pertes totales massiques $P$ en fonction de l'épaisseur e de la tôle [6] (Total losses as a function of sheet thickness) 
La principale exigence concerne l'isotropie de l'induction magnétique et des pertes dans le plan de la tôle puisque l'acier est utilisé dans le champ tournant des moteurs et des générateurs. En pratique, l'isotropie n'est jamais parfaite mais les effets directionnels restent limités, de l'ordre de 10 \% des valeurs de pertes. De ce fait, le critère de qualité des aciers non orientés est représenté par la moyenne des propriétés magnétiques dans la direction de laminage et dans la direction transverse [4].

Les pertes totales massiques diminuent quand l'épaisseur de la tôle décroît (Fig. 4). Elles passent par un minimum, caractérisé par des valeurs de pertes et d'épaisseur de la tôle qui dépendent des conditions de fabrication, notamment de la qualité de l'état de surface [6]. Ce phénomène est particulièrement important pour les tôles de très faibles épaisseurs $(<0,20 \mathrm{~mm})$ utilisées pour les applications à haute fréquence.

Ia quantité, la nature et la morphologie des impuretés présentes dans 1 'acier électrique influencent la valeur des pertes totales massiques. La précipitation lente du carbone et de l'azote en solution solide d'insertion provoque une dégradation des caractéristiques magnétiques du produit final appelée vieillissement [4]. La figure 5 indique l'évolution du vieillissement en fonction de la teneur en carbone des alliages Fe-Si. L'addition de silicium au fer diminue le vieillissement magnétique exprimé sous la forme du quotient de l'augmentation des pertes totales par les pertes totales initiales. L'accroissement du nombre d'impuretés autres que le carbone et l'azote, inclusions de $\mathrm{SiO}_{2}, \mathrm{Al}_{2} \mathrm{O}_{3}, \mathrm{TiO}_{2}$ ou précipités Mns, AIN, formés pendant l'élaboration de l'acier et au cours des traitements thermomécaniques, élève les pertes totales massiques (Fig. 6).
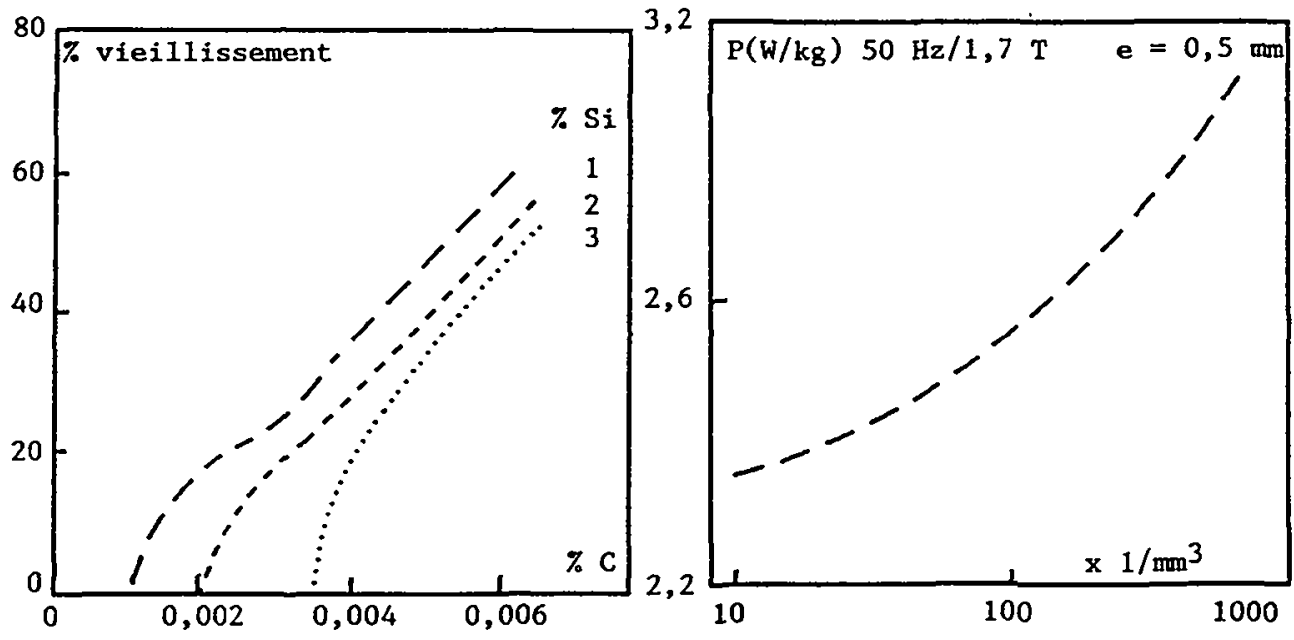

Fig.5 - Influence du carbone et Fig.6 - Influence des impuredu silicium sur le vieillissement magnétique [4] (Effect of $\mathrm{C}$ and $\mathrm{Si}$ on total losses by aging) tés sur les pertes totales massiques [7] (Effect of impurities on total losses) 
Les aciers électriques à grains non orientés ont généralement des grains de diamètre moyen a compris entre 50 et 200 microns. Les pertes totales massiques sont minimales pour un diamètre de grain d'environ 150 microns, quelle que soit la teneur en silicium (Fig. 7). Les impuretés et les joints des grains constituent des obstacles aux déplacements des parois des domaines magnétiques. Quand le diamètre du grain est plus élevé, les pertes par hystérésis générées par les imperfections cristallines diminuent par suite du nombre moins élevé de parois ; mais les pertes dynamiques dues aux courants de Foucault augmentent de par la plus grande vitesse de déplacement des domaines. Ceci explique l'existence d'une taille de grain optimale correspondant au minimum des pertes [4]. Pour favoriser la croissance du grain, il est nécessaire de limiter la quantité d'impuretés $S, 0, N$, Ti qui, à l'état précipité, inhibent cette croissance. A titre d'exemple, la figure 8 montre la relation entre la teneur en $s$ et le diamètre du grain d'un acier à 38Si. Aux teneurs en S élevées, le diamètre du grain plus petit est la conséquence de la présence de fins précipités de Mns.

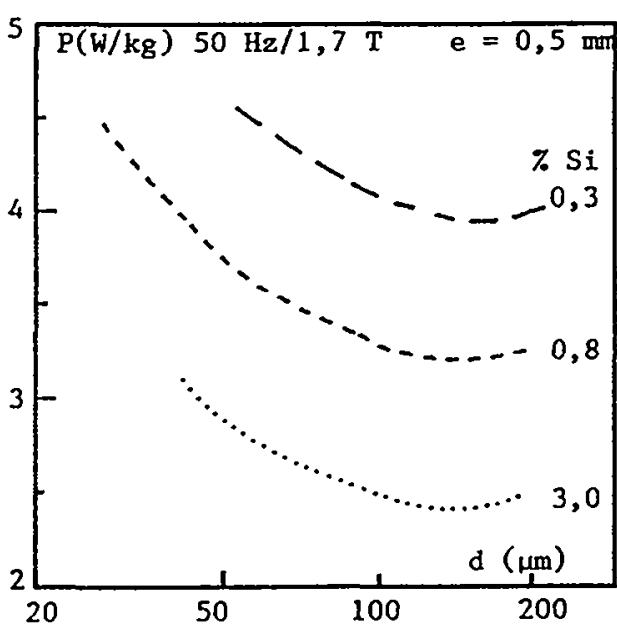

Fig.7 - Influence du diamètre d du grain sur les pertes totales massiques [8]

(Effect of grain size on total losses)

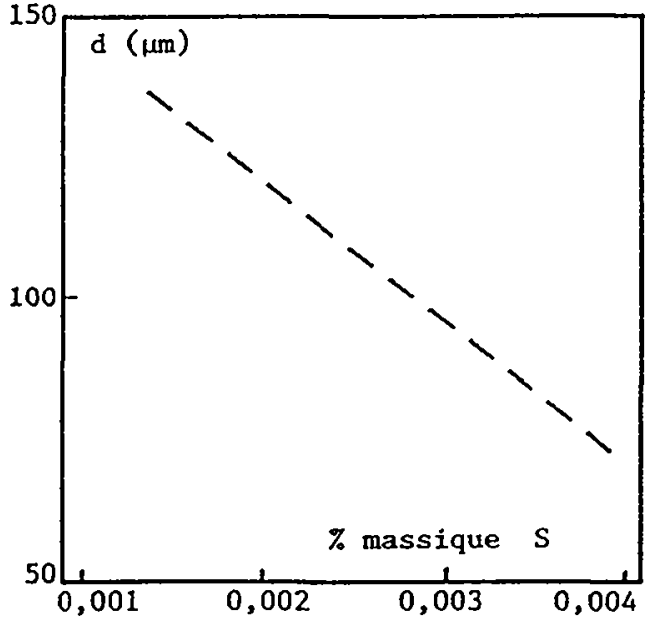

Fig.8 - Influence de la teneur en soufre sur le diamètre d du grain [9]

(Effect of $S$ content on grain diameter)

Deux familles de produits à grains non orientés sont distinguées : les tôles livrées après recuit final et les tôles livrées à l'état semi-fini [4]. Les tôles livrées après recuit final (Fully-Process) possèdent leurs caractéristiques magnétiques garanties par le sidérurgiste à l'état de livraison. Après découpage, un traitement thermique n'est pas nécessaire. Leur choix est avant tout dicté par des considérations plus techniques qu'économiques. Les tôles livrées à l'état semi-fini (Semi-process) ne possèdent pas encore leurs propriétés magnétiques garanties à l'état de livraison par le sidérurgiste. Après découpage, elles doivent subir un traitement thermique indispensable à leur obtention. 
Leur choix est avant tout dicté par des considérations économiques.

\section{Les aciers Fer-Silicium à grains orientés}

Les pertes totales varient en fonction de la désorientation des pertes : elles sont minimales quand la direction de facile aimantation [001] de chaque grain fait un angle $\theta$ voisin de $2^{\circ}$ avec sa projection dans le plan de la tôle (Fig. 9). L'origine de cette variation réside dans l'évolution avec la désorientation de la structure en domaines magnétiques [4].

Les aciers électriques à grains orientés contiennent de 3 à 3,3 \%e silicium. Les différentes étapes de la production sont particulièrement complexes sur les plans technigue et métallurgique [4]. Deux types de tôles à grains orientés sont commercialisées : les tôles classiques et à haute perméabilité. La désorientation moyenne plus faible $\left(3^{\circ}\right.$ au lieu de $\left.7^{\circ}\right)$ des grains de la tôle à haute perméabilité est à l'origine de la différence des pertes existant entre les deux familles de tôles.

La tôle à haute perméabilité acquiert une induction B800 pour un champ de $800 \mathrm{~A} / \mathrm{m}$ (caractéristique de la perfection de l'orientation) pouvant varier de 1,88 à 1,95 T. Le revêtement isolant spécifique de cette tôle a pour effet d'imposer l'équivalent d'une contrainte élastique de traction de 5 à $7 \mathrm{MPa}$ parallèle à la direction de laminage DL (Fig. 10). L'effet de la contrainte induite par un tel revêtement est moins bénéfique vis-à-vis des pertes pour la tôle classique dont la valeur $B 800$ varie de 1,78 à $1,85 \mathrm{~T}$.
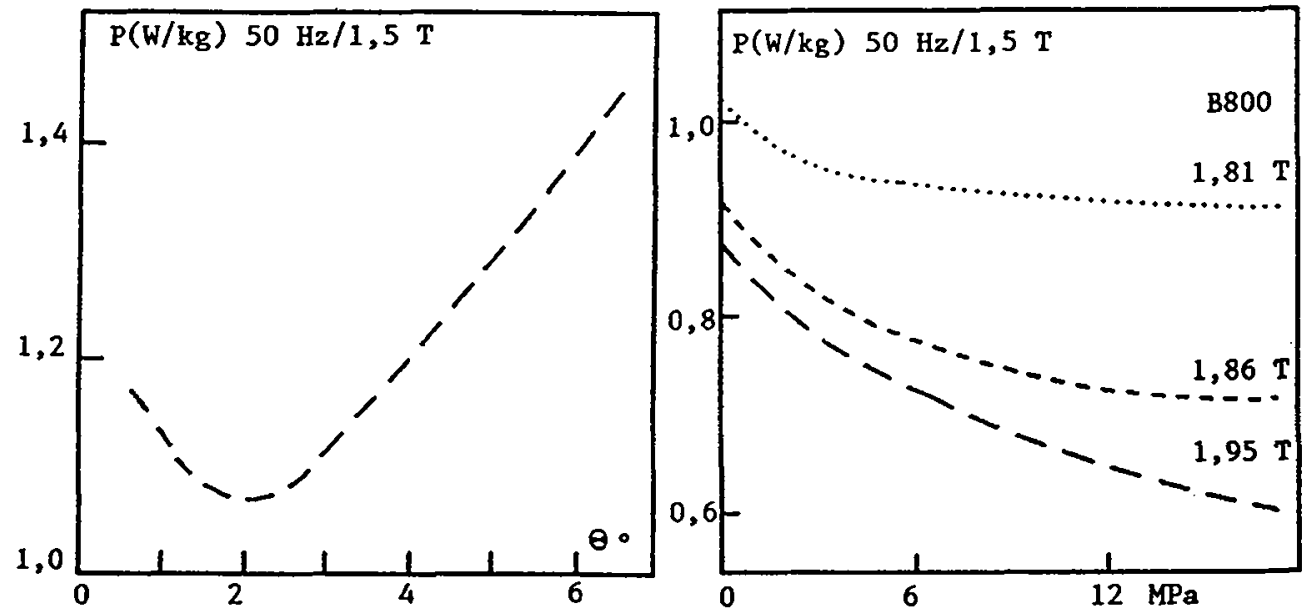

Fig.9 - Variation des pertes totales massiques en fonction de la désorientation [4]

(Effect of misorientation on total losses)
Fig.10 - Influence de l'application d'une contrainte de traction parallèle à DL (Effect of tensile stress applied in the rolling direction)

La variation des pertes totales en fonction de l'épaisseur 
de la tôle passe, comme pour les aciers à grains non orientés, par un minimum qui dépend du degré moyen de désorientation des grains (Fig. 11), de l'état de surface, de l'induction de travail et de la fréquence.

Les épaisseurs disponibles varient respectivement de 0,23 à $0,35 \mathrm{~mm}$ (aciers classiques) et de 0,23 à $0,30 \mathrm{~mm}$ (aciers à haute perméabilité). Les tôles classiques d'épaisseur $0,23 \mathrm{~mm}$ sont commercialisées depuis plusieurs années. Les tôles à haute perméabilité de même épaisseur sont en cours de développement industriel et commercial en Europe. La réduction de l'épaisseur nécessite une mâ̂trise plus grande de l'orientation des grains [10].

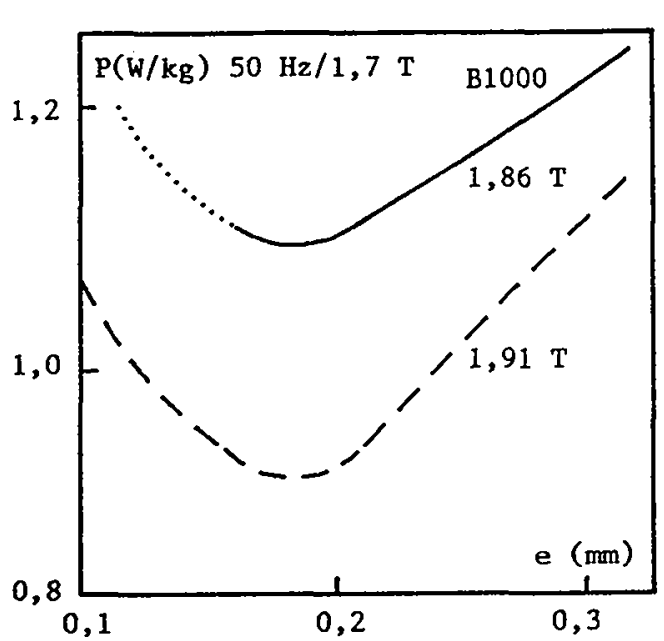

Fig.11 - Pertes totales massiques en fonction de l'épaisseur e [4]

(Total losses as a function of sheet thickness)

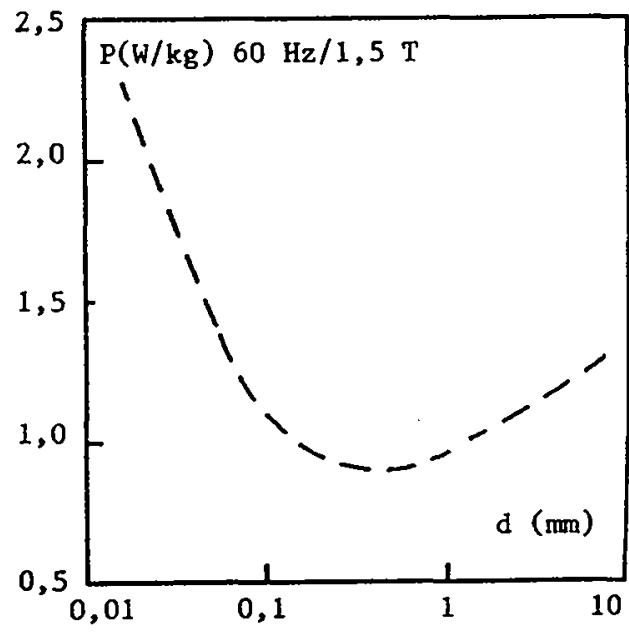

Fig.12 - Pertes totales massiques en fonction du diamètre d du grain [11]

(Effect of grain size on total losses)

Comme pour les aciers à grains non orientés, l'évolution des pertes totales en fonction du diamètre moyen d du grain est caractérisée par l'existence d'un minimum par suite des variations inverses des pertes par hystérésis et des pertes dues aux courants de Foucault (Fig. 12). Le procédé actuel de fabrication des aciers à haute perméabilité génère la formation d'un grain de taille plus grossière $(5$ à $30 \mathrm{~mm})$ que celui des aciers classiques (taille de 1 à $5 \mathrm{~mm}$ ). L'effet néfaste vis-à-vis des pertes de l'existence d'un grain grossier est cependant nettement compensé par l'effet prépondérant d'une meilleure orientation [4].

Les pertes sont corrélées à la structure magnétique des aciers à grains orientés qui est constituée principalement de longs domaines allongés dans la direction de laminage et délímités par des parois à $180^{\circ}$ très mobiles. L'affinement de la structure magnétique consécutif à une augmentation du nombre de domaines aux parois à $180^{\circ}$ est un procédé efficace de réduction des pertes des aciers électriques à haute perméabilité [4]. La technique de l'irradiation de la surface avec un faisceau laser est la plus industrialisée. La figure 13 
montre l'influence d'un traitement laser sur la densité surfacique des domaines à $180^{\circ}$ d'une tôle à haute perméabilité revêtue d'un film isolant induisant une contrainte de traction parallèle à la direction de laminage. Les impacts produits par le balayage du faisceau laser, d'un diamètre et d'un espacement d'environ 200 microns, constituent des lignes parallèles perpendiculaires à la direction de laminage et distantes de 5 $\mathrm{mm}$.
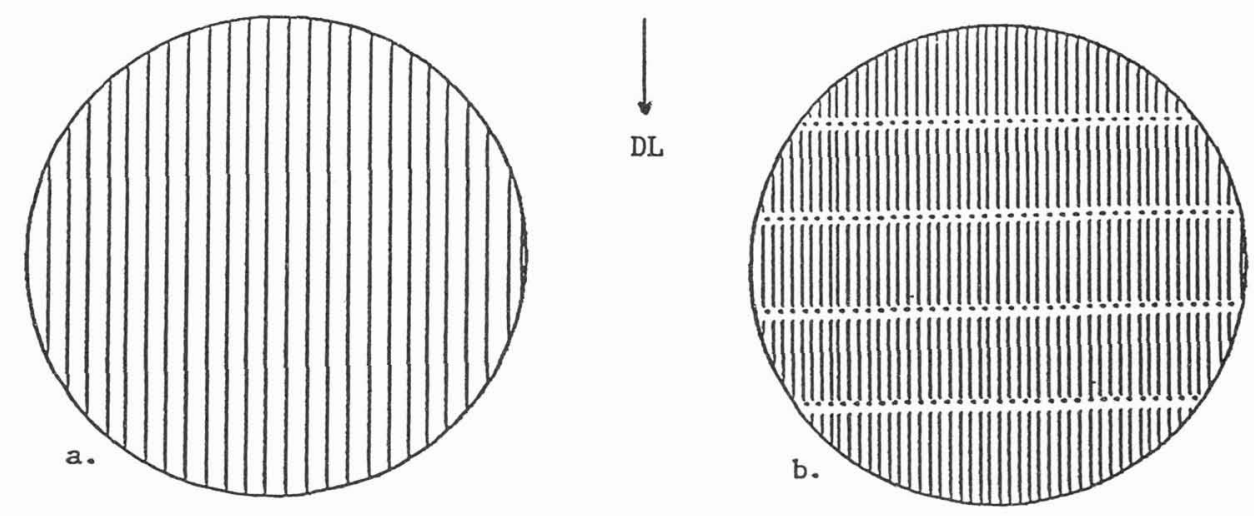

Fig.13 - Schématisation de la structure en domaines d'un acier à haute perméabilité avant (a) et après traitement laser (b) (Appearance of domains before (a) and after (b) laser treatment)

L'efficacité du traitement laser, différence dP entre les pertes avant et après irradiation, est d'autant plus grande que la perfection de l'orientation caractérisée par le B1000 est plus élevée et que l'épaisseur de la tôle est plus faible (Fig. 14).

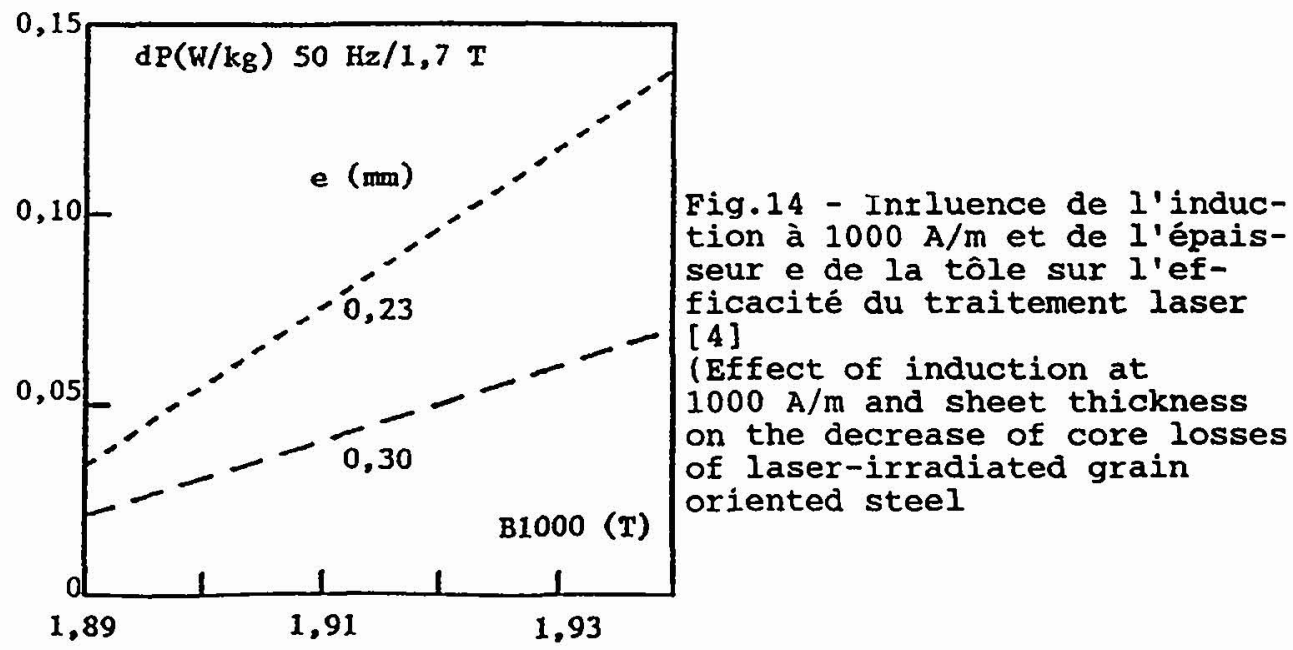


Seules les tôles à haute perméabilité d'épaisseur $0,27 \mathrm{~mm}$ et surtout celles d'épaisseur $0,23 \mathrm{~mm}$ subissent en pratique le traitement laser qui permet d'augmenter le nombre de parois à $180^{\circ}$ qui est lié à la grosseur du grain.

\section{Conclusion : Perspectives d'amélioration des performances} magnétiques

Les aciers électriques à grains non orientés, livrés à l'épaisseur de 0,35 à $0,65 \mathrm{~mm}$, ont des valeurs minimales garanties de $l$ 'induction magnétique pour un champ de $5000 \mathrm{~A} / \mathrm{m}$ qui varient de 1,60 à $1,68 \mathrm{~T}$, les valeurs maximales des pertes totales massiques étant comprises entre 2,50 et $10 \mathrm{w} / \mathrm{kg}$ selon la qualité [4]. Pour les circuits magnétiques des machines tournantes où le flux magnétique est distribué dans des directions variées dans le plan de la tôle, la texture idéale est la texture $\{100\}\langle 0 v w\rangle$ qui donne $l^{\prime}$ induction la plus élevée dans un champ d'excitation donné, grâce à la présence de deux directions de facile aimantation situées dans le plan de la tôle. L'amélioration des performances magnétiques passe donc par la mise au point de procédés favorisant la croissance de grains ayant les plans $\{100\}$ parallèles au plan de la tôle et inhibant la formation de grains ayant les directions de difficile aimantation <111〉 situées dans le plan de la tôle. Il faut, en outre, minimiser l'anisotropie des propriétés magnétiques. I'obtention d'une composante de texture $(100)\langle 0 \mathrm{vw}\rangle$ dans une matrice sensiblement isotrope a été obtenue par certains sidérurgistes, notamment par addition d'éléments d'alliage particuliers ( $\mathrm{Mn}, \mathrm{Sb})$ et recuit de la bande laminée à chaud $[12,13]$. Ugine S.A. a récemment mis au point un procédé original d'obtention d'une texture $\{100\}\langle 0 \mathrm{vw}\rangle$. Le produit, caractérisé par une induction de $1,78 \mathrm{~T}$ pour un champ de 5000 $\mathrm{A} / \mathrm{m}$, est en cours de développement industriel [14]. $L$ 'abaissement de la teneur en éléments résiduels $C, N, 0, S$ grâce à l'élaboration sous vide et à une désulfuration poussée du métal liquide est par ailleurs une autre voie d'amélioration des performances magnétiques des aciers à grains non orientés. outre la réduction des pertes liée à la diminution des obstacles aux mouvements des parois des domaines magnétiques, I'abaissement du niveau des impuretés favorise le grossissement du grain, ce qui n'est pas sans conséquence sur la texture finale. Enfin, la diminution de l'épaisseur des tôles à grains non orientés permettrait de réduire de façon importante les pertes, notamment lorsque la fréquence de travail est supérieure à $50 \mathrm{~Hz}$ [4].

Pour les aciers électriques à grains orientés, la texture idéale est la texture $\{100\}\langle 100\rangle$. En effet, les tôles à grains orientés, qui sont très performantes quand on les utilise parallèlement à la direction de laminage, ont de très mauvaises propriétés magnétiques dans la direction transverse : les pertes sont multipliées par un facteur 3 à 4 et le $B 1000$ diminue d'environ $0,5 \mathrm{~T}$ [4]. L'emploi de tôles à grains orientés $\{100\}\langle 100\rangle$ améliorerait le comportement dans les coins des circuits magnétiques empilés des transformateurs. Cependant, aucun procédé de fabrication d'une telle texture n'a été industrialisé à ce jour, malgré les nombreuses études de laboratoire, en raison des difficultés de maitrise des processus métallurgiques mis en jeu et du prix de 
revient prohibitif de ces produits. Les voies de minimisation des pertes raisonnablement accessibles compte-tenu des contraintes économiques apparaissent être :

- l'amélioration du degré de perfection de la texture

- la diminution de l'épaisseur conjuguée à l'irradiation de la surface par faisceau laser

- l'augmentation de la teneur en silicium (au-dessus de 3,3 8 , I'optimum étant de $6,5 \%$ ), par enrichissement superficiel et traitement de diffusion ou laminage à température supérieure à $100^{\circ} \mathrm{C}$

- l'emploi de tôles à plus faible rugosité de surface obtenues par polissage chimique ou électrochimique avant application du revêtement final.

Il serait illusoire de considérer que les voies possibles de réduction des pertes et d'amélioration de la courbe d'aimantation puissent être développées sans mise en oeuvre des moyens de recherche lourds et coûteux. Il n'est donc pas inutile de rappeler que l'innovation métallurgique $n$ 'a de sens que si les gains réalisés sont effectivement valorisés à leur juste prix.

\section{Bibliographie}

[1] LITIMANN M.F., ASM Sessions on Metallurgy of Magnetic Materials, cleveland, Ohio, 19 Octobre 1970

[2] HALL R.C., J. Appl. Phys. 3 (1960) 1037

[3] PRY R.H., J. Appl. PhYS. 30 (1959) 189-S

[4] BAVAY J.C., VERDUN J., Les Techniques de I'Ingénieur, D2110, D2111, D2112 (1992)

[5] PAD P., CECH D., HORKY P., WIGLASZ V., SMID V., Hutnicke Listy, 43 (1988) 273

[6] GUNTHER K., BOLLING F., HUNEUS H., J. Appl. PhYs., 64 (1988) 5347

[7] MATSUMARA K., FUKUDA B., KINOSHITA K., IMAI T., OBATA Y., MIYAZAKI S., Kawasaki Steel Technical Report, 8 (1983) 11

[8] SHIOZAKI $M$. et KUROSAKI Y., J. Materials Engineering, 11 (1989) 37

[9] MATSUMARA K., FUKUDA B., IEEE Transactions on Magnetic, 20 (1984) 1533

[10] BAVAY J.C., Revue Générale de l'Electricité, 11 (1989) 32

[11] DEGAUQUE J., Mém. et Et. Sc. Rev. Mét., 1 (1985) 5

[12] SHIOZAKI M., KUROSAKI Y., Textures and microstructures, 11 (1989) 159

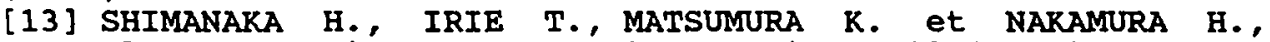
Journal of Magnetism and Magnetic Materials, 19 (1980) 63

[14] BAVAY J.C., VERDUN J., Symposium on Hard and Soft Magnetic Materials with Applications, 1-5 Nov. 1992, Chicago 